\title{
Successful visceral revascularization after acute type A aortic dissection: How to predict visceral ischemia
}

\author{
Manabu Shiraishi, Kouichi Adachi, Hiroshi Nagano, Atsushi Yamaguchi and Hideo Adachi \\ Department of Cardiovascular Surgery, Saitama Medical Center, Jichi Medical University, Japan
}

\begin{abstract}
We report a case of a 64-year-old woman with acute aortic dissection who developed visceral ischemia after ascending aortic graft replacement. After primary surgery, serum lactate and base excess levels were suddenly increased. Enhanced computed tomography showed interrupted celiac artery perfusion. Emergent common hepatic artery and right common iliac artery bypass grafting was performed. The patient recovered without resection of abdominal organs. Visceral ischemia should be kept in mind as a possible complication in patients with acute aortic dissection, and this complication can be expected by monitoring of base excess and lactate serum level.
\end{abstract}

\section{Introduction}

Acute aortic dissection with visceral ischemia is potentially a fatal complication after acute aortic dissection. Early diagnosis and surgical treatment are required for rescue irreversible abdominal organ necrosis [1-4]. Here we describe a successful case of visceral revascularization and avoidance of abdominal organ necrosis after the onset of acute Stanford type A aortic dissection.

\section{Case report}

A 64-year-old woman presented with severe back pain and the absence of left femoral artery pulse. Enhanced computed tomography (ECT) demonstrated a type A aortic dissection entry at the ascending aorta. The true lumen gradually became smaller in diameter, and expanded false lumen compressed the true lumen of the celiac artery and interrupted the celiac artery perfusion. The superior mesenteric artery and both renal arteries originated from the true lumen, and the dissection was extended to left external iliac artery (Figure 1). Emergent ascending aortic graft replacement was performed. Six hours after the primary surgery, serum lactate levels abruptly increased to 7.53 $\mathrm{mmol} / \mathrm{L}$, although the value lowered to $4.32 \mathrm{mmol} / \mathrm{L}$, postoperatively. Simultaneously, base excess serum levels rapidly increased from -2.5 to $-9.5 \mathrm{mmol} / \mathrm{L}$. Other laboratory data including plasma creatine kinase level, C-relative protein level and liver enzyme levels were acceptable at this time. At the same time, motor and sensory dysfunctions in both legs were observed. We strongly suspected visceral ischemia and paraplegia due to interruption of innate vessel perfusion. Repeat ECT demonstrated that the severe constriction of the true lumen of the celiac artery resulting from compression by the expanded false lumen presented earlier remained unchanged (Figure 2). An emergent operation was performed for visceral ischemia. We planed the common hepatic artery and right common iliac artery bypass grafting by using the great saphenous vein. The color of the stomach and liver was pale; however, ascites was not identified. The great saphenous vein was harvested, and the common hepatic artery and right common iliac artery were exposed. The right common iliac artery-common hepatic artery bypass was performed through the mesocolon transversum. Soon after bypass grafts, the color of stomach and liver changed to red, and the base excess serum level and the lactate levels were turned normalized immediately. Spinal drainage and management of systemic blood pressure gradually improved paraplegia.

The patient's recovery was uneventful. Postoperative ECT demonstrated patency of the graft (Figure 3). After 1 month, this patient is in good condition without abdominal angina and paraplegia.

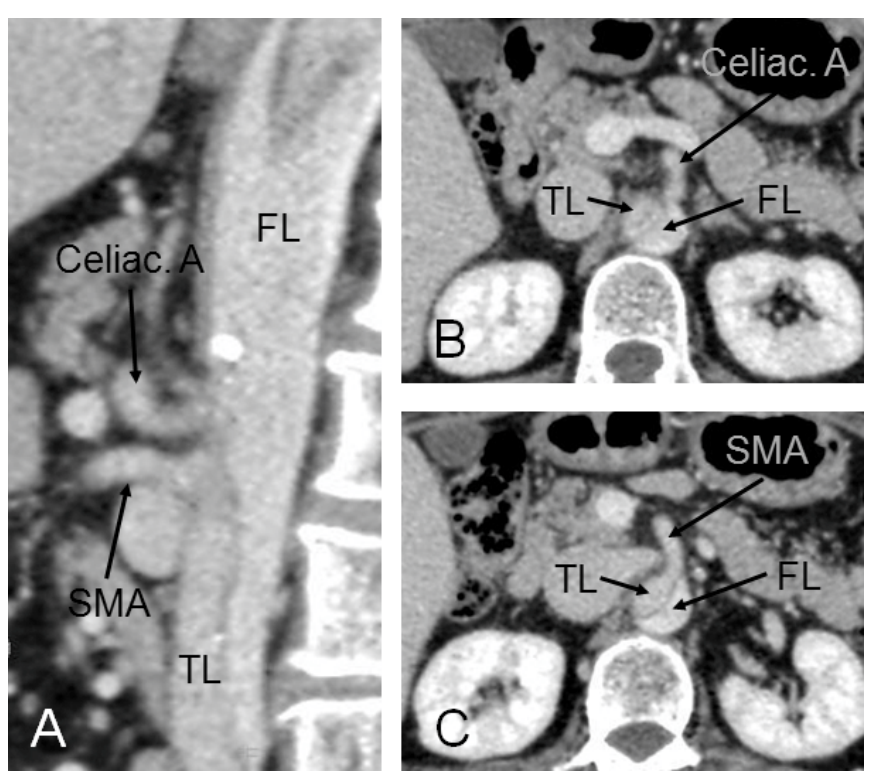

Figure 1. Sagittal view of preoperative enhanced computed tomography showing the interrupted celiac artery perfusion from true lumen (A). The celiac artery (B) and the superior mesenteric artery $(\mathrm{C})$ were perfused from false lumen and true lumen, respectively. $S M A$, superior mesenteric artery; $T L$, true lumen; $F L$, false lumen.

Correspondence to: Manabu Shiraishi, Division of Cardiovascular Surgery, Saitama Medical Center, Jichi Medical University, Amanuma, Omiya-Ku, Saitama-City, Japan, Tel: +81-48-647-2111; Fax: +81-48-648-5188; E-mail: manabu@omiya.jichi.ac.jp

Key words: acute aortic dissection, visceral ischemia, visceral revascularization

Received: November 27, 2017; Accepted: December 26, 2017; Published: December 29, 2017 


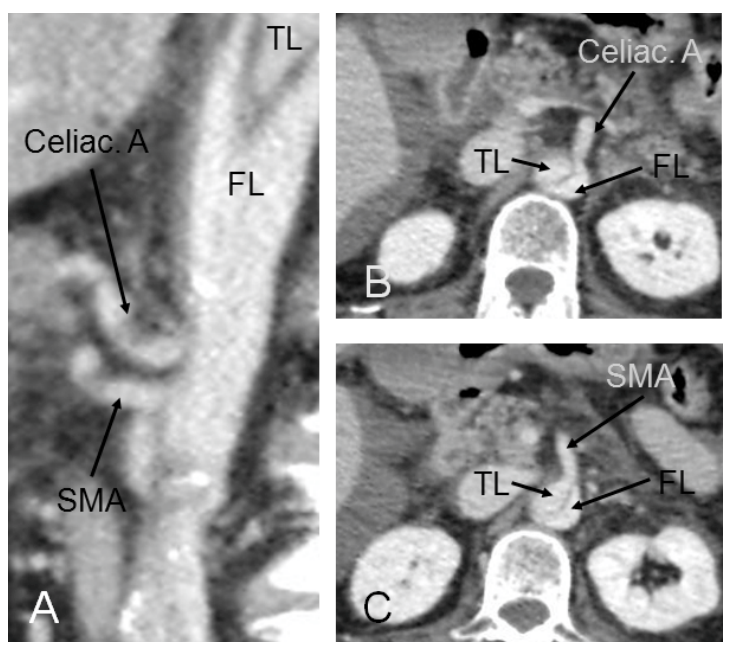

Figure 2. Sagittal view of postoperative enhanced computed tomography showing the unchanged structure of true and false lumen compared with those of the preoperative period (A). The celiac artery (B) and the superior mesenteric artery (C) were perfused from false lumen. $S M A$, superior mesenteric artery; $T L$, true lumen; $F L$, false lumen.

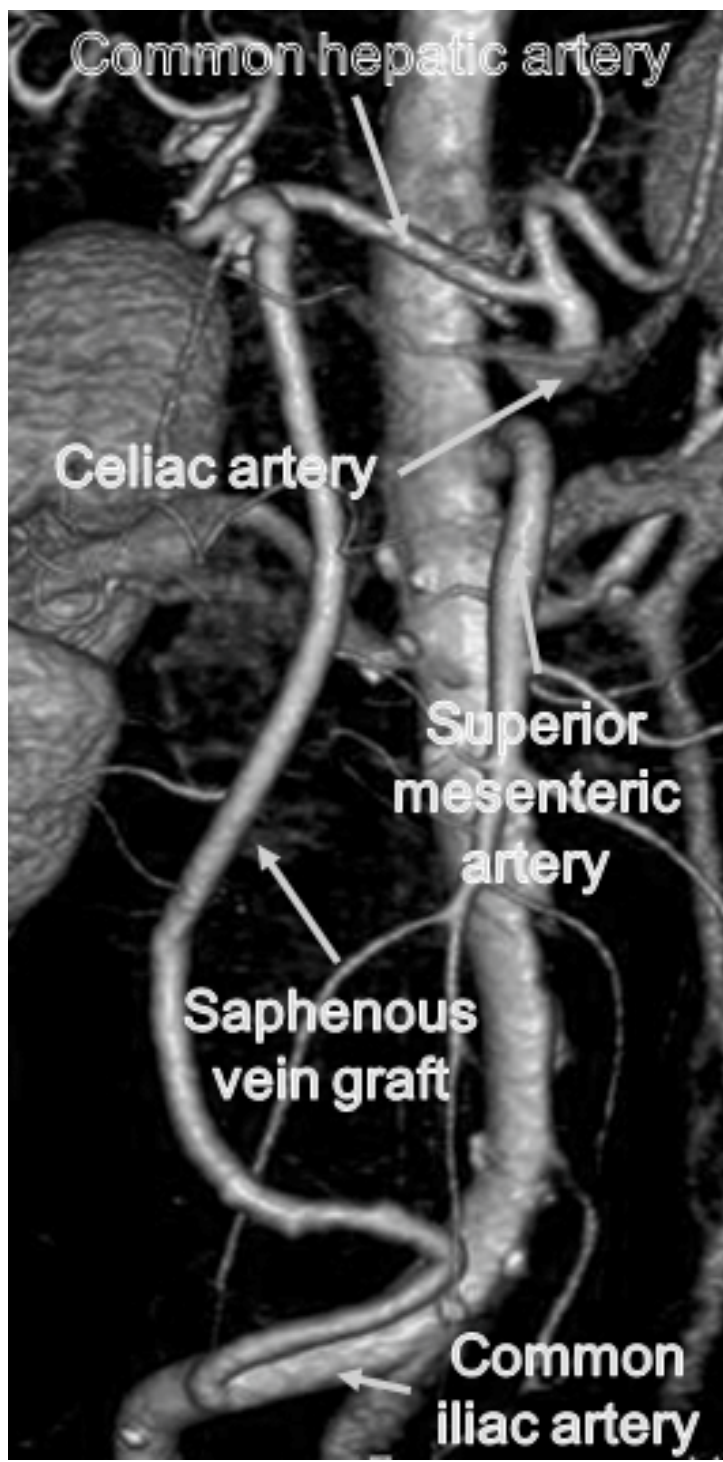

Figure 3. 3-dimensional enhanced computed tomography showing that saphenous vein graft was patent.

\section{Discussion}

Visceral ischemia complicated by acute aortic dissection, which increase the overall risk of aortic dissection, occurred as a complication in $<5 \%$ of patients with acute aortic dissection, and mortality of patients with malperfusion was 40\% [1-3]. Although the outcomes of acute aortic dissection treatment have improved, visceral ischemia is still a serious problem because there are no clear diagnosis criteria [1]. Visceral ischemia with aortic dissection in previous reports were often diagnosed by symptoms, blood examination (transaminase, creatine phosphokinase, and, ammonia levels), computed tomography, magnetic resonance angiography, ultrasonography and laparoscopy $[1,3]$. Although advanced necrosis of intestines is easily diagnosed, inadequate visceral artery perfusion resulting in abdominal organ necrosis is difficult. Furthermore, visceral artery perfusion that is consistent as apparently intact portions may become ischemic later, depending on unexpected changes in perfusion by primary aortic surgery. In our case, entry resection and ascending aortic replacement did not change the form of true and false lumen in the celiac artery orifice, and celiac artery perfusion did not improve.

Lactate, accumulated in prolonged bouts of interrupted blood flow, has been reported to be a sensitive serum marker for early diagnosis of acute visceral ischemia [3-5]. In our case, cautious observation at fixed intervals of the base excess serum level and lactate level were effective to detect inadequate visceral artery perfusion, and eventually visceral organ resection was avoided. Therefore, especially in patients with a severely narrowed true lumen of the visceral branch vessels, visceral perfusion should be evaluated by continuous blood examination primarily.

Bypass grafting from the intact iliac artery to common hepatic artery with a saphenous vein graft is a common procedure. Fenestration of the intimal flap in the aorta and implantation of a covered stent, which have been reported as favorable results equal to surgical treatment, are possible alternative treatments for visceral ischemia [1].

\section{Conclusion}

To avoid a delay in appropriate timing of treatment, monitoring of base excess and lactate serum level and prompt revascularization treatment are desirable.

\section{Disclosure statement}

No external funding was obtained for the work presented here. The authors have no conflicts of interest to declare.

\section{References}

1. Orihashi K (2016) Mesenteric ischemia in acute aortic dissection. Surg Today 46: 509516. [Crossref]

2. Heinemann MK, Buehner B, Schaefers HJ, Jurmann MJ, Laas J, et al. (1994) Malperfusion of the thoracoabdominal vasculature in aortic dissection. J Card Surg 9: 748-755. [Crossref]

3. Stone JR, Wilkins LR (2015) Acute mesenteric ischemia. Tech Vasc Interv Radiol 18: 24-30. [Crossref]

4. Muraki S, Fukada J, Morishita K, Kawaharada N, Abe T (2003) Acute type A aortic dissection with intestinal ischemia predicted by serum lactate elevation. Ann Thorac Cardiovasc Surg 9: 79-80. [Crossref]

5. Günel E, Cağlayan O, Cağlayan F (1998) Serum D-lactate levels as a predictor of intestinal ischemia-reperfusion injury. Pediatr Surg Int 14: 59-61. [Crossref]

Copyright: (C2017 Shiraishi M. This is an open-access article distributed under the terms of the Creative Commons Attribution License, which permits unrestricted use, distribution, and reproduction in any medium, provided the original author and source are credited. 